\title{
COMPUTERIZED SURGICAL PLANNING OF THE OSTEOCONDORAL TRANSPLANT WITH HELP OF PATIENT SPECIFIC GUIDANCE INSTRUMENTS
}

\author{
Theodor Cezar Milian'1, Nicoleta Mirela Popa ${ }^{2}$, Nichita Larisa Milodin ${ }^{3}$ \\ 1,2,3Research Assistant, Mechatronics-Biomedical-Robotics Department, \\ National Institute of Research and Development in Mechatronics and Measurement Technique \\ miliantheodor@yahoo.com,popa_nicoleta2093@yahoo.com,nichita.milodin@gmail.com
}

\begin{abstract}
A successful restoration of the articular cartilage by osteochondral transplantation depends on positioning the transplanted cylindrical grafts as precisely as possible in order to reconstruct the curvature and the height of the original articular surface. This experimental study shows a computerized method for achieving pre-surgical planning of transplantation and correct positioning of the osteochondral cylindrical grafts, using patient-specific guides designed and manufactured by rapid prototyping techniques available in the country.

The computerized method consists in the computerized reconstruction of the 3D virtual bone model and the adjacent cartilage using the patient's medical data provided by CT images, MRI, etc., in the development of a computerized plan for harvesting grafts from the donor sites and in placing them on the site of the defect with the aid of customized guides.

Guides are designed with specialized 3D reconstruction programs from CT, MRI (eg MIMCS) images so that the bottom surface of the image is perfectly matched to the contour of the articular surface of the bone. On the upper surface there are cylindrical bodies in which guiding holes are provided for precise positioning of the surgical instruments for extracting and inserting osteochondral grafts. The guiding tools were designed for a male patient to be used on his left knee.

Results. Preoperative planning based on 3D virtual anatomical models and using the patientspecific guidelines for grafting and insertion of the grafts provide good results in positioning and orientating them, allowing a high-precision reconstruction of the joint surface in a short time, with no need of optically targeted intraoperative guidance.
\end{abstract}

Keywords: Mosaicplasty, Patient-Specific Instrument Guides, Computer-Assisted Mosaicplasty, CT Data, Mimics, 3D CAD Model, Rapid Prototyping, Selective Laser Sintering, PA 2200.

\section{Introduction}

Articular cartilage is a smooth tissue that covers the ends of a joint's bones in order to make the movement easier by allowing them to slide over each other with very little friction. It can be damaged by trauma or normal wear. Cartilage degeneration is a widespread problem that occurs predominantly in the knee, ankle and shoulder. Since the articular cartilage is not vascularized, once it is damaged it can not be repaired naturally. Mechanical damage to the surfaces of the cartilage leads to long-term damage and loss of joint surface [1]. The treatment of cartilaginous and osteocartilaginous lesions is one of the major problems of modern orthopedics. On the one hand, the endoprosthetic treatment of this pathology presents several disadvantages, particularly for young people, whose lifetime of endoprostheses is shorter than those of older patients.
Despite that, because the damaged cartilage is not vascularize, it has a limited repair potential through spontaneous regeneration, and despite that, when such repair of a lesion occurs, it is partial and the repaired tissue is a fibrocartilage with mechanical properties far inferior to hyaline cartilage.

Taking into account all this and due to the high frequency of cartilaginous and osteocartilaginous lesions encountered in over the years, the problem of regeneration and repair of cartilaginous surfaces has become more and more relevant, in this sense several surgical techniques has been developed. of these, the only technique that performs hyaline cartilage repair is osteochondral transplantation, recognized as mosaic arthroplasty.

This surgical technique consists of harvesting several osteochondral cylindrical grafts from a donor area (a non-articulating area of the joint) and implanting them in the osteochondral defect $[2,3]$ (see Figure 1) 

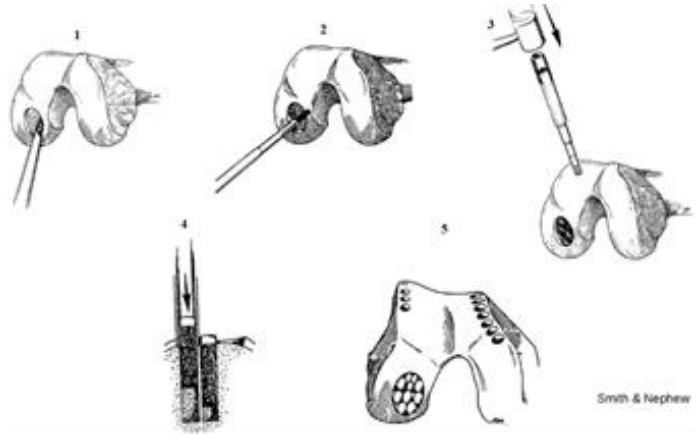

Figure 1: 5-step mosaic arthroplasty procedure (after Smith and Nephew): 1) removal of debris; 2) machining of holes for the insertion of osteochondral cylindrical grafts; 3) graft harvesting from nonimportant areas; 4) insertion of osteochondral grafts, 5) final result

From a technical point of view, mosaic arthroplasty is a difficult operation that requires a lot of precision.

Although this surgical technique is frequently applied, clinical trials in osteochondral transplant patients have shown that it is very important to create a congruent articular surface. Besides that, due to poor accessibility of donor sites and varying radius of curvature of joint surfaces specific to each patient it is difficult to duplicate a congruent joint surface when using multiple small grafts. Concerning these difficulties, the surgical treatment that consists in reconstruction of osteochondral cartilage by autologous or allograft graft transplantation remains a valuable reconstruction option for cartilage defects, but the precision of graft harvesting and insertion remains problematic. To achieve the objectives of an ideal arthroplasty, osteochondral grafts harvested from a variety of donor joints must match the natural curves of the site of the host site specific to each patient. The osteochondral cylindrical grafts should be inserted so as they come to rebuild the initial articular surface. The upper surface of grafts harvested from a donor site (nonarticulating surface area) is curved and may not be perpendicular to the axis of the donor site. Thus, the positioning and orientation of each graft must be planned for it to be achieved. Grafts that are too large can lead to poor integration due to micromotion and increased contact pressure [4,5]. Grafts that are too small can cause cartilage necrosis and increase excess fibrocartilage with mechanical properties far inferior to hyaline cartilage. [6]. Also, if a graft with upper angular (inclined) surface is incorrectly inserted, it may be too high on either side of the original surface and on a too-buried part. If the reconstructed surfaces are higher than the original surface, the quality of healing decreases a lot [7]. Computerized planning and intraoperative guidance are used to solve these problems. The surgical plan indicates the position and orientation of the sites for harvesting and inserting each osteochondral cylindrical graft. During surgery, the surgeon follows the plan using patient-specific guides [8].

The process varies from patient to patient, depending on the degeneration's degree. Mainly, after establishing the defect zone level, it is necessary to CAD design a virtual $3 \mathrm{D}$ model of a guide for the patient-specific sampling and inserting tools, whose lower surface is identical to the articular surface subjected to osteochondral transplantation.

The computer-assisted surgical plan consisted of:

1. Reconstruction of the original articular joint surface;

2. Evaluation of cartilage thickness in donor and receptor areas so that the thickness of the reconstructed cartilage matches the initial thickness of the cartilage to avoid degeneration at the bone/cartilage interface;

3. Planning the donor sites so that the orientation of the surface of each osteochondral graft matches with the anticipated orientation of the surface of the recipient site;

4. Design an optimal mosaic template (model) to ensure defect coverage with minimum overlapping and spacing.

Pre-chronic planning has proven to be effective in finding harvesting and delivery sites for reconstruction of the original joint surface [8]. This requires a highly skilled human operator with knowledge of mosaic arthroplasty and the ability to use a 3D computer interface.

\section{Related Work}

Various clinical assessments have shown the importance of reconstructed surface congruence for cartilage repair. In an animal study [4], it was shown that grafts that were too high were diminished by weight but showed cracks, bone cysts, and poor bone incorporation. Grafts with inclined surfaces (parts of these surfaces positioned above the surrounding surface) are subject to high contact pressure [5], resulting in maximum loading and abrasion of the surface of the graft [10]. Nevertheless, grafts that have been inserted too deeply can be disposed of due to inadequate pressure [11] and showed a necrosis of cartilage and an excessive increase in fibrocartilage [6]. Planning and computerized navigation have been successfully used in the retrographic insertion of grafts into the human talus [12]. Planning consisted of using patient images to manually select the trajectory for the surgical drill. Another study using talus found out that harvesting and insertion of grafts are better done accomplished using the computer [13]. Computer assisted planning has also been used to match grafts harvested from femoral conditions with thalus defects [14]. 


\section{CAD Modeling of the Anatomical Model}

This paper describes a methodology applied in developing a virtual 3D model of a distal femur in CT medical data using the Mimics software. The result of this virtual modeling is useful for computerized surgical planning of osteochondral transplantation and for the patient-specific guide needed for precise positioning and orientation of grafts during surgery.

The detailed anatomical 3D model of the knee was created using medical data provided by a set of standard DICOM images scanned using computed tomography (CT). The DICOM images generated in CT scan were then processed with Mimics Research software 18.0.0.525 to obtain the primary 3D model using density segmentation techniques (see Figure 2).

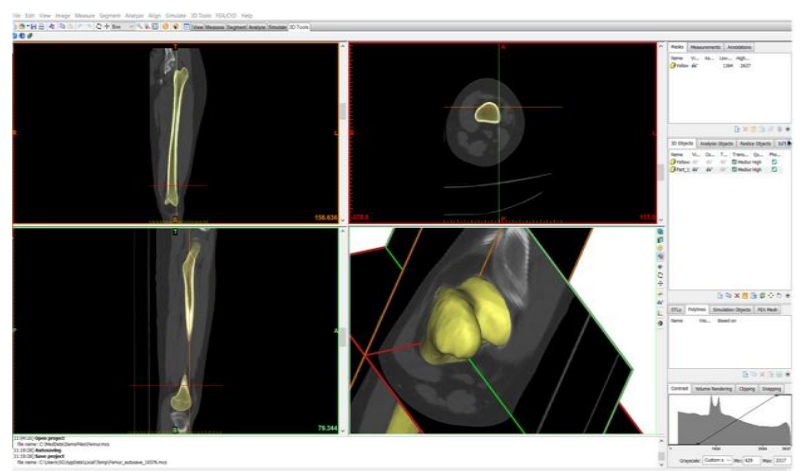

Figure 2: Virtual 3D model reconstruction from medical images of a pacient's femur

The 3D generated primary models were then processed and assembled as geometric data files (see Figure 3). Finally, the model was exported as a .stl file.

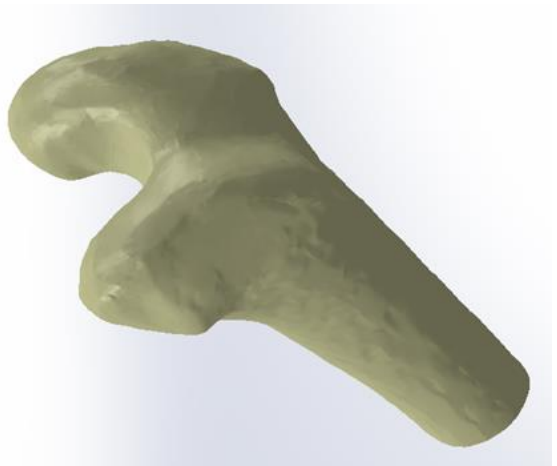

Figure 3: Distal femur 3D model

The medical image segmentation software Mimics18.0.0 was used to reconstruct the 3D model. The separate $3 \mathrm{D}$ reconstruction of each bone segment was performed with manual editing of the density masks.

\section{Pre-Operative Planning}

On the 3D surfaces of the distal femur was created a surgical consisting plan of a set of osteochondral cylindrical grafts positioned above the defect site (see Figure 4).

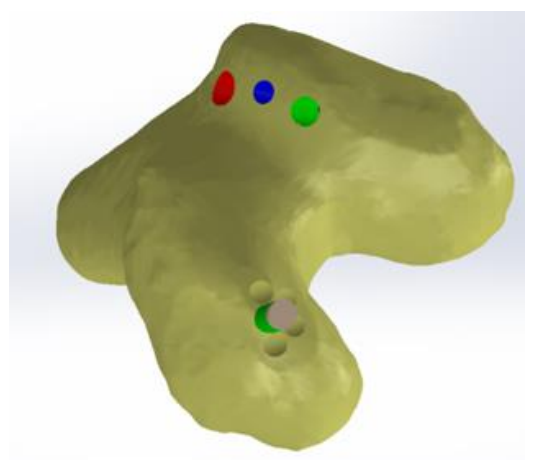

Figure 4: Planning the grafts to rebuild the defective surface

The 3D position and orientation of each graft, as well as its shape (diameter, height and slope) were chosen to best rebuild the desired joint surface at the site of the defect (see Figure 5).
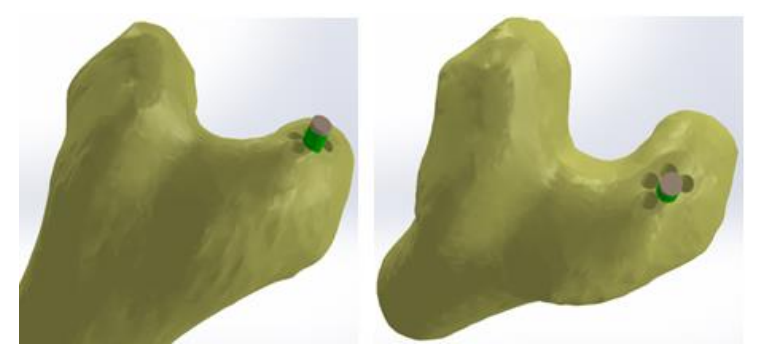

Figure 5: Positioning and graft orientation in the receptor site

For each graft, a donor site was selected to ensure the best fit with its shape. The grafts can be rotated axially so that the inclined surface of the harvesting site is best matched to the inclined surface of the defect site to obtain the best congruence of the joint surface (see Figure 6).

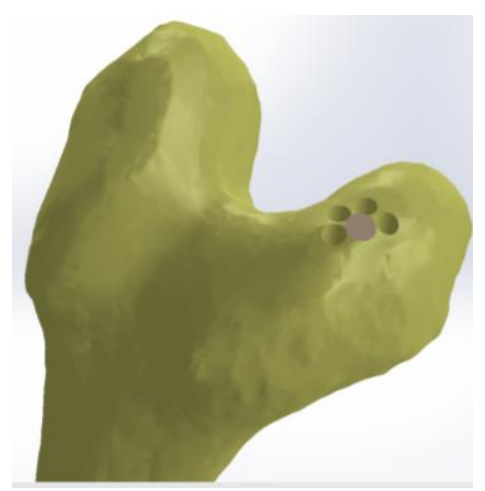

Figure 6: Adaptation of the surface of the graft to the surrounding surface 
The surgical plan is validated by overlapping grafts on 3D anatomical models.

\section{Construction of the Patient-Specific Guide}

For each patient, a set of personalized guides can be constructed, containing a tagging guide, a collection guide, and an insertion guide for each scheduled graft. The lower surface of each guide is designed to fit exactly with the knee joint surface (see Figure 7) in the area for which it is intended.

Thus, the planned position of the guide can be correctly reproduced intraoperatively by adjusting its position until an exact match with the surface of the cartilage is achieved. Each guide is built on an EOS FORMIGA P110 Rapid Prototyping Machine by Selective Laser Sintering (SLS), PA2200 Biopolymer Polyamide Powder.

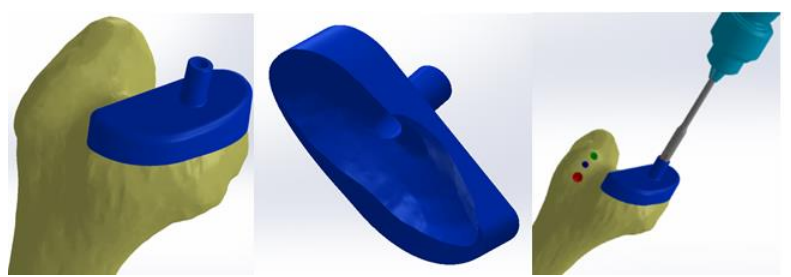

Figure 7: Patient-specific guidelines for graft insertion and harvesting

The tagging guide is designed to fit exactly with the area of the harvesting area (the donor site), and for each graft contains a hole corresponding to the planned harvesting site. Each hole is provided with a circumferential marker in relation to which the surgeon draws a radial line on the surface of the cartilage to follow the axial rotation of the graft.

The harvesting guide is designed to fit exactly with the harvest joint surface and contains a guiding drum for the harvesting instrument of each graft. Also, the height of the guide rollers can be made in such a way as to ensure the penetration of the harvesting instrument up to the planned depth.

The insertion guide is designed to fit exactly with the insertional joint surface (site of the defect) and contains a single graft guide cylinder. This guide is used to guide the borehole during drill holes as well as to guide the graft insertion tool. The rotation mechanisms in the guide holes ensure that the harvested graft is inserted with the correct alignment with respect to its axis.

\section{Intra-Surgical Procedure}

After the incision is performed, the marking guide is positioned on the knee joint surface in the sampling area and a reference mark is made for the rotation of each graft. Then the harvesting guide is positioned exactly on the harvest area and fixed with the Kirscher plugs.

Using the guiding cylinders, grafts are harvested using cutting tools. The length of each graft can be viewed through the slits practiced in the harvest/ insertion tools and can be checked with the gradations on these instruments. For each graft harvested, insert the guide on the surface of the defect and process the receiver tunnel with a drill. Then, the graft is inserted by means of the insertion tool into the guide roller so that the grading rotation mark on the graft is aligned with a corresponding rotation mark on the guide roller. After aligning the two markings, the graft is pushed into the receiver tunnel and the insertion guide specific to this graft is removed to resume the insertion procedure of another graft.

\section{Conclusions}

Using 3D CAD modeling software for different bones of the skeleton allows someone to generate the most complex joint surfaces of virtual anatomical models, based on which it can be created templates, guides, and other tools needed to make a pre-surgical plan. The above results have shown that process of making the virtual anatomical model using Mimics requires a short time to create the model from the patient's CT medical data. Based on the 3D virtual model of the distal femur from the knee joint, someone can observe some significant advantages:

- Creating a very complex anatomical model requires a very little amount of time;

- The 3D model created can be created with different materials if biomechanical bone simulation is required;

- The shorter duration of the design/ manufacturing cycle of patient-specific surgical guidelines;

- 3D CAD modeling of human bones with specialized programs is useful for kinematic analysis of joints by finite element analysis. The 3D models of the guidelines described above are used for computer assisted planning of osteochondral transplantation in the treatment of cartilaginous defects for implant surgery in case of total knee replacement, but also for manufacturing using rapid prototyping techniques.

With the help of 3D CAD set guides to fit exactly with the articular surface of the cartilage, we developed a computer-assisted surgical arthroplasty planning method that provides better coverage of the defective area and is more precise than classical surgery. Computer-aided planning assures a lower graft overlay, a shorter distance between the graft and surgery with patient-specific guidelines requires less time than the classic, even if performed by an experienced surgeon. 
Clinical trials conducted using optical guidance techniques or template guidance techniques have shown that both techniques have had a significantly greater effect than conventional surgical treatment [15].

Guided techniques can cause a slight friction in the moment the graft is inserted, thus reducing the penetration of synovial fluid because the drill is held rigidly inside the guide cylinder while preparing the tunnel receptors in the defect area. With conventional techniques, all surgical instruments are portable, without external support, and can lead to reception tunnels and grafts that can present some deviation from circularity.

However, further studies are needed to confirm that this improvement in cartilage reconstruction by osteochondral transplantation assisted by patientspecific guidelines will translate into a better longterm clinical outcome.

\section{References}

[1] Inoue J, Kunz M, Hurtig MB, Waldman SD, Stewart AJ.,Automated planning of computer assisted mosaic arthroplasty,Med Image Comput Comput Assist Interv. 2011;14(pt1):267-74.

[2] Yamashita, F.: The transplantation of an autogeneic osteochondral fragment for osteochondritis dissecans of the knee. Clin Orthop Relat Res (201) (1985) 43

[3] Hangody, L., Kish, G., Krpti, Z., Szerb, I., Udvarhelyi, I.: Arthroscopic autogenous osteochondral mosaicplasty for the treatment of femoral condylar articular defects: A preliminary report. Knee Surgery, Sports Traumatology, Arthroscopy 5(4) 262-

[4] Pearce, S., Hurtig, M., Clarnette, R., Kalra, M., Cowan, B., Miniaci, A.: An investigation of 2 techniques for optimizing joint surface congruency using multiple cylindrical osteochondral autografts. Arthroscopy: The Journal of Arthroscopic and Related Surgery 17(1) (2001) 50-55

[5] Koh, J., Kowalski, A., Lautenschlager, E.: The effect of angled osteochondral grafting on contact pressure: A biomechanical study. American Journal of Sports Medicine 34(1) (2006) 116-119

[6] Huang, F., Simonian, P., Norman, A., Clark, J.: Effects of small incongruities in a sheep model of osteochondral autografting. American Journal of Sports Medicine 32(8) (2004) 1842-1848
[7] Kunz, M., Hurtig, M., Waldman, S., Devlin, S., Rudan, J., Bardana, D., Stewart, J.: Image-guided surgical techniques for cartilage repair - an animal trial. In: World Congress of the International Cartilage Repair Society (ICRS). (2010) 190

[8] Radermacher, K., Portheine, F., Anton, M., Zimolong, A., Kaspers, G., Rau, G., Staudte, H.W.: Computer assisted orthopaedic surgery with image based individual templates. Clin Orthop Relat Res (354) (September 1998) 28-38

[9] Kunz, M., Devlin, S., Rudan, J., Waldman, S., Stewart, J., Hurtig, M.: Computerassisted planning for mosaic arthroplasty. In: Computer Assisted Radiology and Surgery. (2009) S102-S103

[10] Jakob, R., Franz, T., Gautier, E., Mainil-Varlet, P.: Autologous osteochondral grafting in the knee: indication, results, and reflections. Clinical Orthopaedics and Related Research 401 (2002) 170-184

[11] Koulalis, D., Benedetto, P.D., Citak, M., OLoughlin, P., Pearle, A., Kendoff, D.: Comparative study of navigated versus freehand osteochondral graft transplantation of the knee. American Journal of Sports Medicine 37(4) (2009) 803-807

[12] Bale, R., Hoser, C., Rosenberger, R., Rieger, M., Benedetto, K., Fink, C.: Osteochondral lesions of the talus: Computer-assisted retrograde drilling - feasibility and accuracy in initial experiences. Radiology 218 (2001) 278

[13] Hoser, C., Bichler, O., Bale, R., Rosenberger, R., Rieger, M., Kovacs, P., Lang, T., Fink, C.: A computer assisted surgical technique for retrograde autologous osteochondral grafting in talar osteochondritis dissecans (ocd): a cadaveric study. Knee Surgery, Sports Traumatology, Arthroscopy 12(1) (2004) 6571

[14] Marymount, J., Shute, G., Zhu, H., Varner, K., Paravic, V., Haddad, J., Noble, P.: Computerized matching of autologous femoral grafts for the treatment of medial talar osteochondral defects. Foot and Ankle Intl 26(9) (2005) 708

[15] [Manuela Kunz, Steven M. Devlin, Mark B. Hurtig, Stephen D. Waldman, John F. Rudan, Davide D. Bardana, and A. James Stewart ImageGuided Techniques Improve the Short-Term Outcome of Autologous Osteochondral Cartilage Repair Surgeries - An Animal Trial. Cartilage. 2013 Apr; 4(2): 153-164. 